\title{
Aqueous Photochemistry of 2-Methyltetrol and Erythritol as Sources of Formic Acid and Acetic Acid in the Atmosphere
}

\author{
James D. Cope, ${ }^{1}$ Karizza A. Abellar, ${ }^{2}$ Kelvin H. Bates,${ }^{1,3}$ Xuan Fu, ${ }^{1}$ Tran B. Nguyen ${ }^{1 *}$
}

1. Department of Environmental Toxicology, University of California Davis, Davis, California 95616, United States

2. Department of Chemistry, University of California Davis, Davis, California 95616, United States

3. Center for the Environment, Harvard University, Cambridge, Massachusetts 02138, United States

* Correspondence to: $\underline{\text { tbn@ } \mathrm{ucdavis.edu}}$

Pages: 11

Figures: 4

Tables: 3

Schemes: 1

Mechanisms: 1 


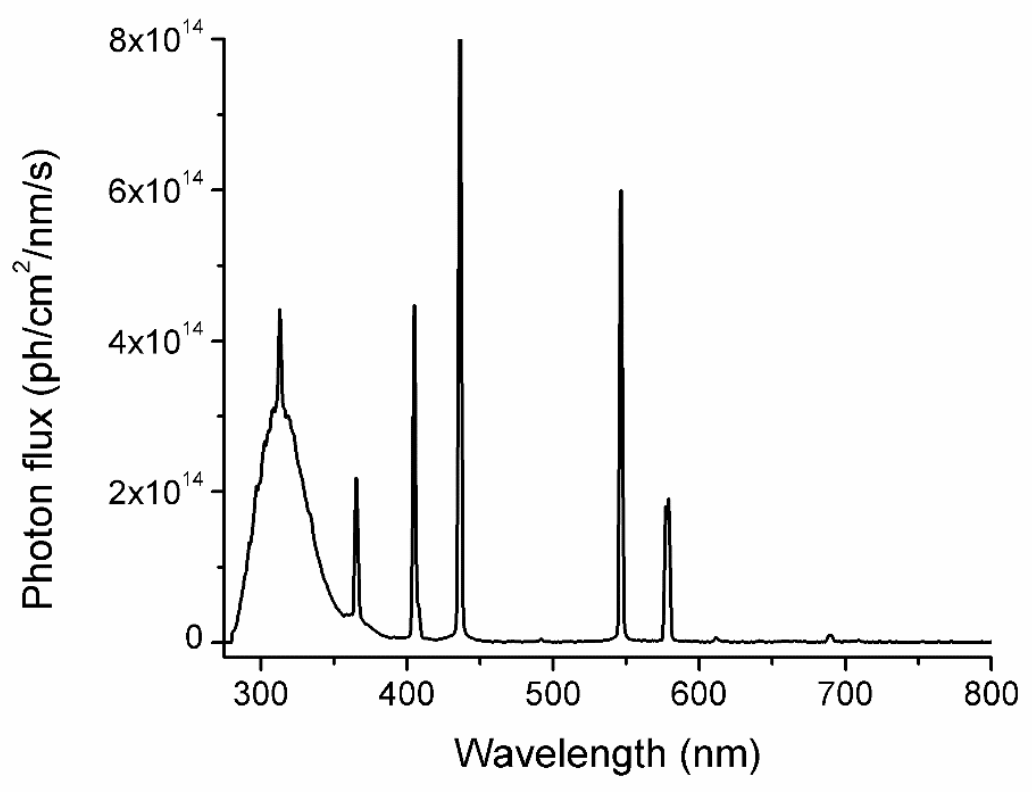

Figure S1 Emission photon flux of UV-B broadband fluorescent light from the photooxidation chamber.

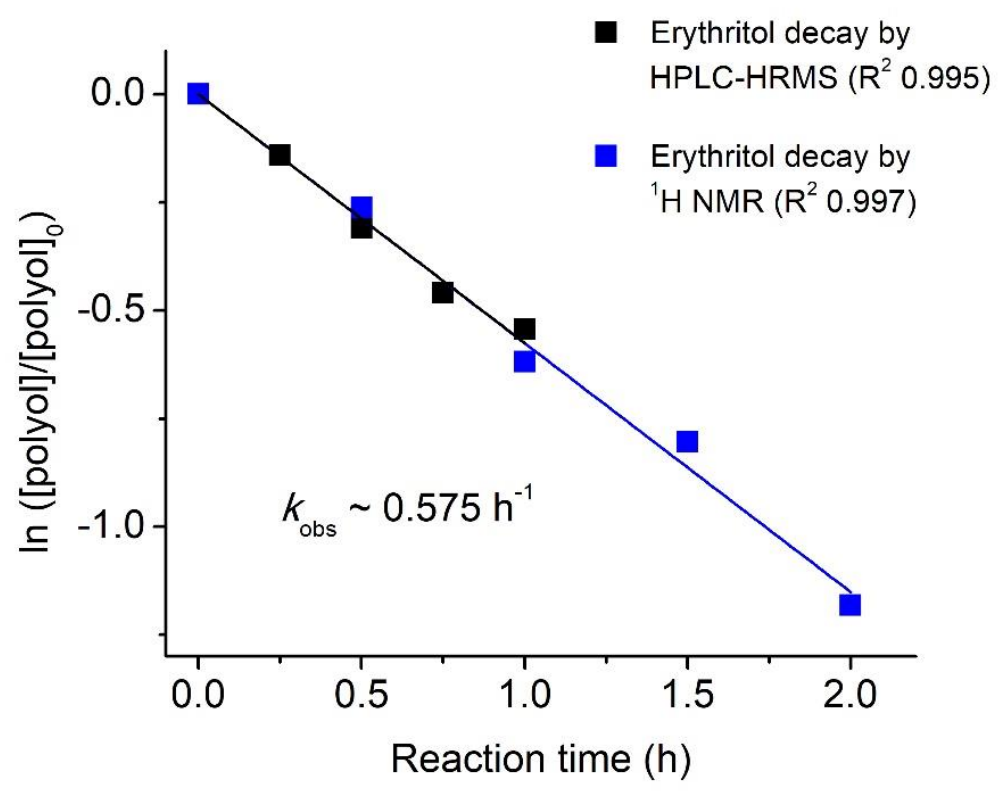

Figure S2 Pseudo first order kinetic decay of erythritol in the $\mathrm{OH}$ oxidation reaction as monitored by HPLC-HRMS and NMR. 


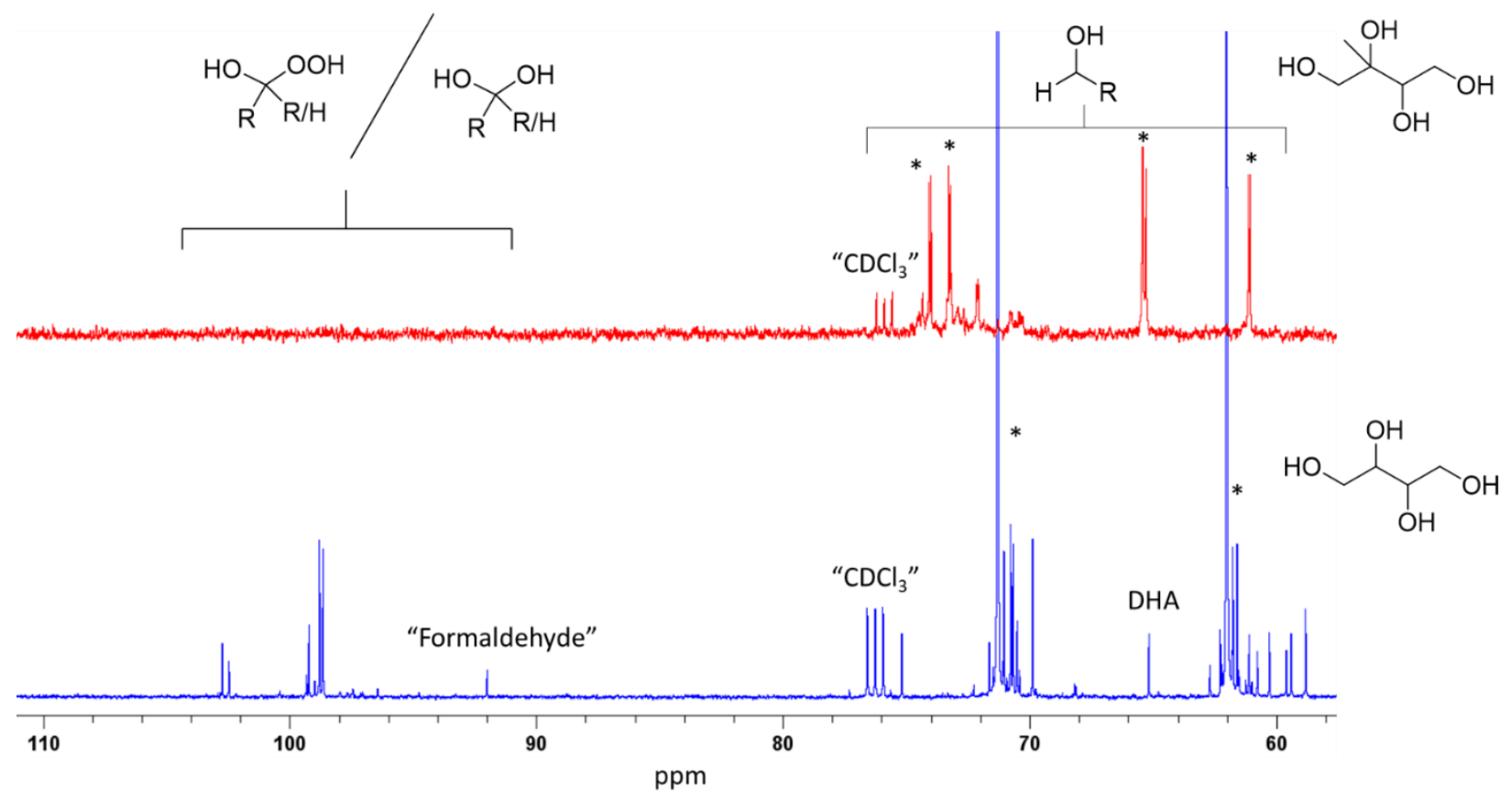

Figure S3 Expanded view of the $60-110$ ppm region in the ${ }^{13} \mathrm{C}$ NMR spectra of the $2-\mathrm{MT}+\mathrm{OH}$ (red) and $\mathrm{E}+\mathrm{OH}$ reaction (blue) after 30 minutes of reaction. 


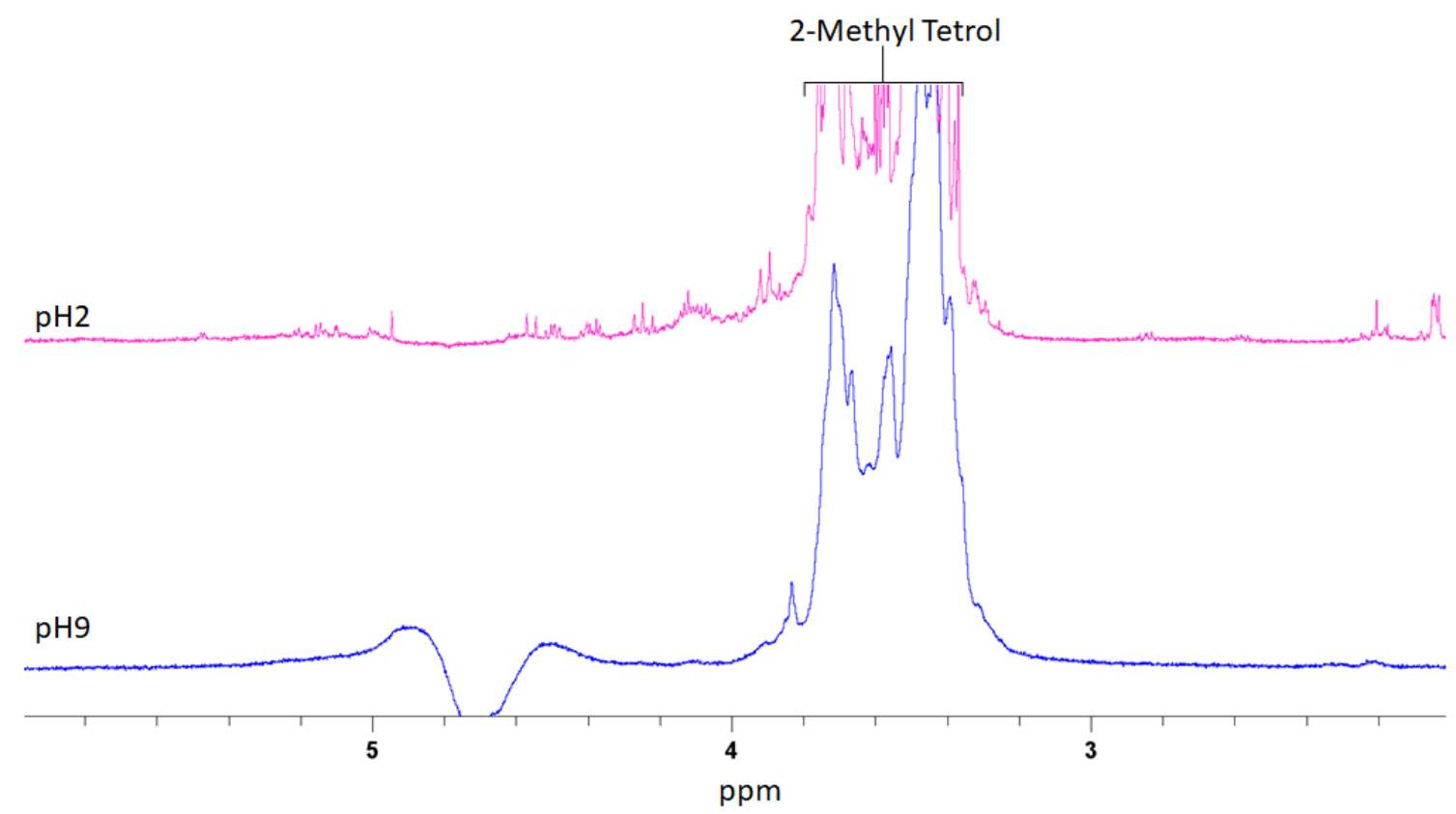

Figure S4 Magnification of the 2-6 ppm region in the $1 \mathrm{H}$ NMR spectra for $2-\mathrm{MT}+\mathrm{OH}$ at $\mathrm{pH} 2$ (magenta) and $\mathrm{pH} 9$ (blue) after 1.5 hours of reaction time, performed with water suppression, showing intermediate products. Asterisks denote hydrogens belonging to the parent polyol. 
Table S1 Identified proton signals in the ${ }^{1} \mathrm{H}$ NMR of the erythritol $+\mathrm{OH}$ reaction.

\begin{tabular}{|c|c|}
\hline Signal $(\delta \mathrm{ppm})$ & Compound \\
\hline 8.21 & \\
\hline 4.20 & \\
\hline 2.08 & \\
\hline 5.01 & $\mathrm{HO}$ \\
\hline $2.13,4.36$ & \\
\hline $2.24^{\mathrm{a}}$ & \\
\hline $2.67^{\mathrm{a}}$ & \\
\hline
\end{tabular}

${ }^{\mathrm{a} U n c o n f i r m e d}$ 
Table S2 Observed DNPH-adduct ions, molecular formulas of the associated carbonyl/acid, and assigned structures for the molecular formulas in the reaction of erythritol $+\mathrm{OH}$. Significant peaks with signal to noise $>5$ are shown in bold.

\begin{tabular}{lll}
\hline $\begin{array}{l}\text { Observed } \\
\text { Mass }\end{array}$ & Molecular Formula & Possible Structure $(\mathrm{s})^{\mathrm{a}}$ \\
$(\mathrm{m} / \mathrm{z})$ & & \\
\hline $\mathbf{2 0 9 . 0 2 8}$ & $\mathrm{CH}_{2} \mathrm{O}$ &
\end{tabular}

225.023 $\mathrm{CH}_{2} \mathrm{O}_{2}$<smiles>O=CO</smiles>

$237.023 \quad \mathrm{C}_{2} \mathrm{H}_{2} \mathrm{O}_{2}$<smiles>O=CC=O</smiles>

$239.038 \quad \mathrm{C}_{2} \mathrm{H}_{4} \mathrm{O}_{2}$<smiles>CC(=O)O</smiles>

$255.033 \quad \mathrm{C}_{2} \mathrm{H}_{4} \mathrm{O}_{3}$<smiles>O=C(O)CO</smiles>

269.049 $\quad \mathrm{C}_{3} \mathrm{H}_{6} \mathrm{O}_{3}$<smiles>O=CC(O)CO</smiles>

281.05 $\quad \mathrm{C}_{4} \mathrm{H}_{6} \mathrm{O}_{3}$<smiles>C=C(O)C(O)C=O</smiles><smiles>O=C(/C=C/O)CO</smiles>

(dehydrated $\mathrm{C}_{4} \mathrm{H}_{8} \mathrm{O}_{4}$ )

$285.043 \quad \mathrm{C}_{3} \mathrm{H}_{6} \mathrm{O}_{4}$<smiles>O=C(O)C(O)CO</smiles>

$297.044 \quad \mathrm{C}_{4} \mathrm{H}_{6} \mathrm{O}_{4}$<smiles>O=CC(O)C(=O)CO</smiles><smiles>O=CC(O)C(O)C=O</smiles> 
$299.059 \quad \mathrm{C}_{4} \mathrm{H}_{8} \mathrm{O}_{4}$
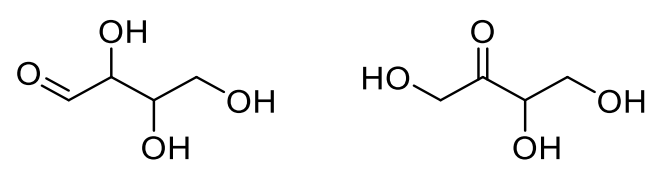

$301.053 \quad \mathrm{C}_{3} \mathrm{H}_{6} \mathrm{O}_{5}$

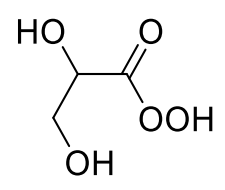

$313.037 \quad \mathrm{C}_{4} \mathrm{H}_{6} \mathrm{O}_{5}$<smiles>O=CC(O)C(O)C(=O)O</smiles>

$315.055 \quad \mathrm{C}_{4} \mathrm{H}_{8} \mathrm{O}_{5}$

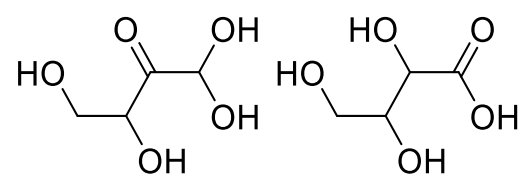

${ }^{a}$ Expected structure prior to DNPH derivatization 
Table S3 Observed DNPH-adduct ions, molecular formulas of the associated carbonyl/acid, and assigned structures for the molecular formulas in the reaction of 2-methyltetrol $+\mathrm{OH}$. Significant peaks with signal to noise $>5$ are shown in bold.

\begin{tabular}{lll}
\hline $\begin{array}{l}\text { Observed } \\
\text { Mass }\end{array}$ & Molecular Formula & Possible Structure $(\mathrm{s})^{\mathrm{a}}$ \\
$(\mathrm{m} / \mathrm{z})$ & & \\
\hline $\mathbf{2 0 9 . 0 2 8}$ & $\mathbf{C H}_{2} \mathbf{O}$ & O $_{\mathrm{H}}$
\end{tabular}

$225.023 \quad \mathrm{CH}_{2} \mathrm{O}_{2}$<smiles>O=CO</smiles>

223.047 $\mathrm{C}_{2} \mathrm{H}_{4} \mathrm{O}_{2}$<smiles>CC=O</smiles>

239.038 $\quad \mathrm{C}_{2} \mathrm{H}_{4} \mathrm{O}_{2}$<smiles>CC(=O)O</smiles>

251.042 $\quad \mathrm{C}_{3} \mathrm{H}_{4} \mathrm{O}_{2}$

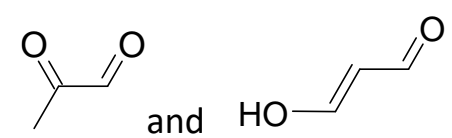

$253.057 \quad \mathrm{C}_{3} \mathrm{H}_{6} \mathrm{O}_{2}$<smiles>CC(=O)CO</smiles>

$255.033 \quad \mathrm{C}_{2} \mathrm{H}_{4} \mathrm{O}_{3}$<smiles>O=C(O)CO</smiles>

265.057 $\mathrm{C}_{4} \mathrm{H}_{6} \mathrm{O}_{2}$<smiles>CC(=O)C(C)=O</smiles>

$269.053 \quad \mathrm{C}_{3} \mathrm{H}_{6} \mathrm{O}_{3}$<smiles>O=CC(O)CO</smiles>

$281.052 \quad \mathrm{C}_{4} \mathrm{H}_{6} \mathrm{O}_{3}$

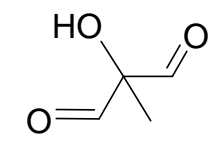


$283.068 \quad \mathrm{C}_{4} \mathrm{H}_{8} \mathrm{O}_{3}$

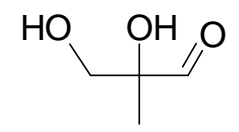

297.047 $\mathrm{C}_{4} \mathrm{H}_{6} \mathrm{O}_{4}$

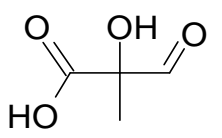

$313.037 \quad \mathrm{C}_{5} \mathrm{H}_{10} \mathrm{O}_{4}$

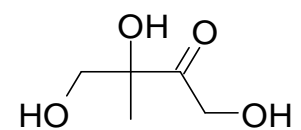

$327.058 \quad \mathrm{C}_{5} \mathrm{H}_{8} \mathrm{O}_{5}$

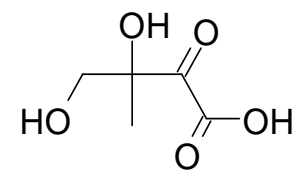

${ }^{a}$ Expected structure prior to DNPH derivatization 

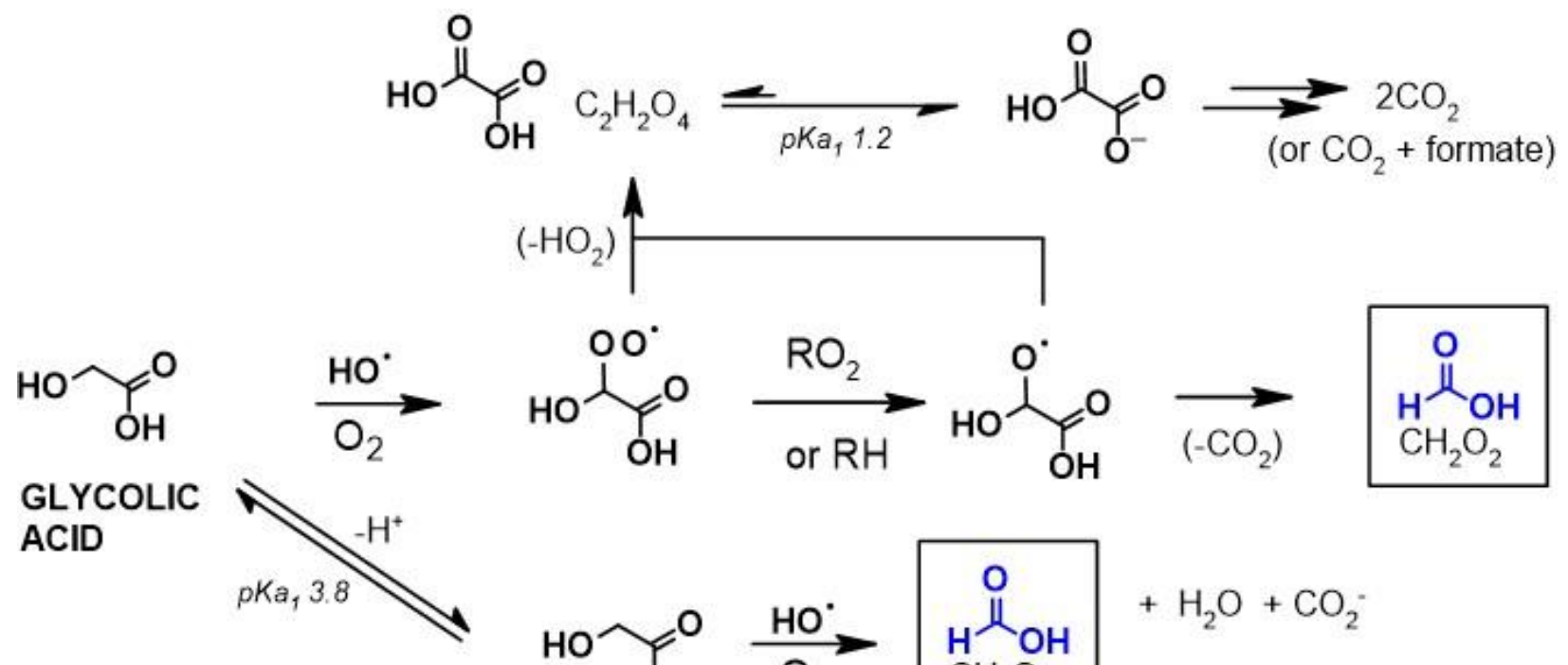

GLYCOLIC ACID

Scheme S1 Proposed OH oxidation reaction of glycolic acid in the aqueous phase. 
Mechanism S1 Simplified model mechanism for the formation of formic acid (FA), acetic acid (AA), and their carboxylates from the $\mathrm{OH}$ oxidation of 2-methyltetrol (2-MT) at pH 2 and pH 9. The mechanism requires input of light flux (Fig. S1), photolysis of $\mathrm{H}_{2} \mathrm{O}_{2}$, and known $\mathrm{HO}_{\mathrm{x}}$ reactions $\left(\mathrm{OH}+\mathrm{H}_{2} \mathrm{O}_{2}, \mathrm{HO}_{2}+\mathrm{HO}_{2}, \mathrm{OH}+\mathrm{HO}_{2}\right.$, JPL Evaluation 2016) in order to initiate the $\mathrm{OH}$ radical production.

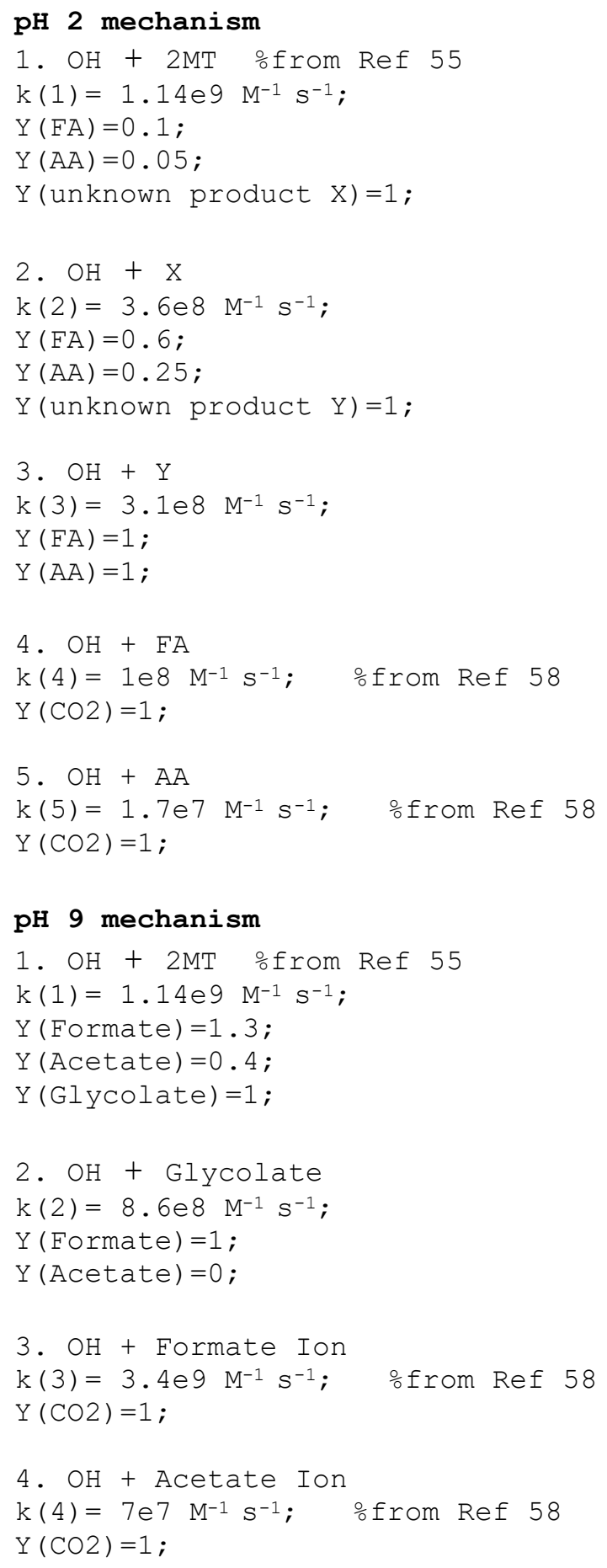

